\section{A la recherche de l'essence} de I'humour

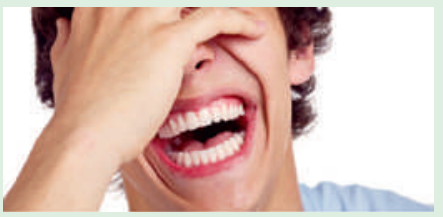

Lorsqu'une personne rit d'une blague, un processus à deux étapes s'opère dans son cerveau. Celui-ci discerne d'abord une contradiction avant de la transformer en joie. Les processus cérébraux liés à la compréhension de l'humour ne sont pas les mêmes chez les filles et chez les garçons. C'est l'une des conclusions à laquelle est arrivé un scientifique basé aux EtatsUnis et soutenu par le Fonds national suisse.

(Fonds national suisse de la recherche scientifique)

\section{Welche Ausbildungs- alternativen wählen abgewiesene Medizin- studenten?}

Nur vier von zehn Kandidaten, die sich für ein Medizinstudium einschreiben, kennen das Studienangebot zu den Gesundheitsberufen an den Fachhochschulen. Damit Gesundheitsberufe als Alternative zum Medizinstudium häufiger berücksichtigt werden und der Fachkräftemangel im Gesundheitswesen der Schweiz gemindert wird, braucht es gemäss einer ZHAW-Studie im Auftrag des Bundesamts für Gesundheit BAG mehr Studienplätze. Die Gymnasiastinnen und Gymnasiasten sollten frühzeitig über das ganze Spektrum an Berufen im Gesundheitswesen informiert werden. Universitäre und FachhochschulStudiengänge sollten dabei gleich behandelt werden.

(ZHAW Zürcher Hochschule für Angewandte Wissenschaften)

\section{Grenzwerte für Feinstaub schützen ungenügend vor Sterberisiko}

Feinstaubkonzentrationen in der Luft, die weit unter den geltenden EU- und Schweizer Grenzwerten liegen, verursachen bedeutend mehr Todesfälle als angenommen. Das zeigt eine neue gesamteuropäische Studie von über 360000 Bewohnern grosser Städte in 13 europäischen Ländern. Bereits eine Vergrösserung der Langzeitbelastung von 5 Mikrogramm Feinstaub (PM2.5) pro Kubikmeter Luft im Jahresmittel, erhöht das natürliche Sterberisiko um 7\%. Das entspricht etwa dem Unterschied an Luftverschmutzung zwischen einem städtischen und einem eher ländlichen Wohnort der Schweiz. «Die heutigen EU-Grenzwerte für Feinstaub sind zu hoch. Sie schützen nicht vor chronischen Krankheiten und ihren Risiken», sagt Prof. Nino Künzli vom Schweizerischen Tropenund Public Health-Institut (Swiss TPH).

(Swiss TPH)

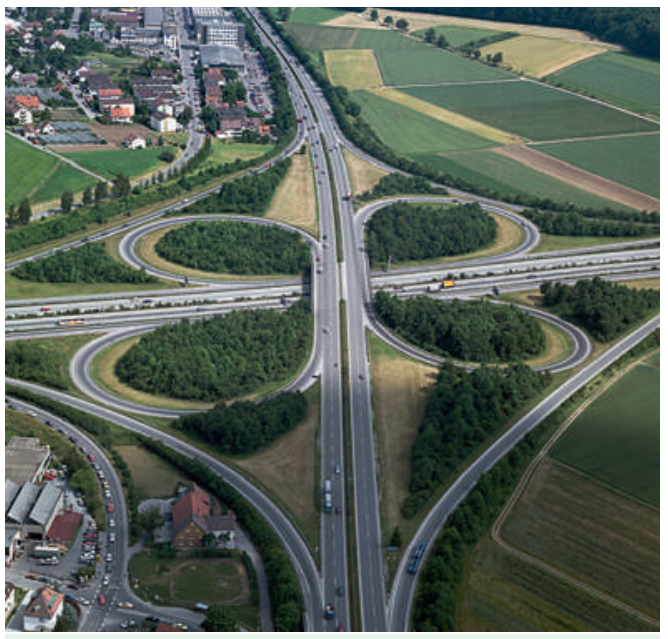

Schützen die geltenden Richtwerte für Feinstaubkonzentrationen ausreichend vor Gesundheitsschäden?

\section{Rétrospective sur la politique en matière d'alcool}

Inscrite dans diverses lois, la prévention structurelle en matière d'alcool est en pleine évolution.

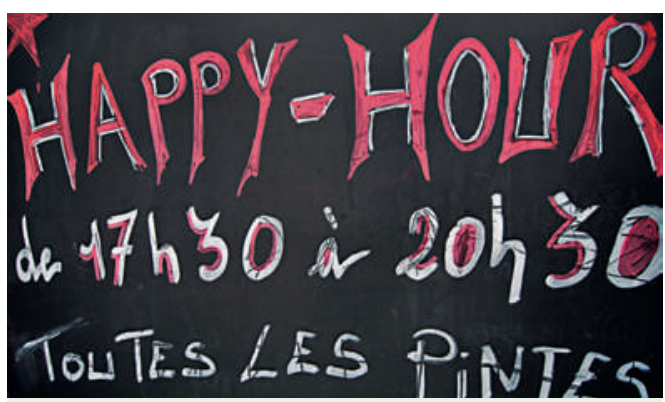

Addiction Suisse plaide pour une approche sociétale qui ne renvoie pas la responsabilité de la consommation problématique à l'individu.

Le défi politique est de définir la responsabilité sociale plutôt que de reporter sur l'individu la gestion du problème. En effet, face aux intérêts de l'industrie de l'alcool, il est extrêmement difficile de faire passer les grandes mesures visant à réduire les consommations problématiques. Au terme de sa révision totale, la loi sur l'alcool aura une influence déterminante sur les prochaines générations. Celui qui boit trop et se retrouve à l'hôpital sera lui-même responsable de sa situation. Addiction Suisse plaide pour une approche sociétale qui ne renvoie pas la responsabilité de la consommation problématique d'alcool au seul individu, mais qui la place dans le cadre de la collectivité.

(Addiction Suisse)

\section{Veranstaltung: Zeitvorsorge}

Vitale und rüstige Menschen im 3. Lebensabschnitt sollen hochbetagten und gebrechlichen Menschen im 4. Lebensalter helfen. Dafür erhalten sie Zeitgutschriften, eine konjunkturunabhängige Währung, die sie dann später selber einlösen können, wenn ein Pflege- oder Hilfebedarf eintritt. Diese Idee klingt einfach und ist auf den ersten Blick bestechend, enthält Chancen und Risiken. Es gibt aber auch eine kritische Diskussion: Sollen der Staat, die Kantone oder die Gemeinden in funktionierende freiwillige soziale Unterstützungssysteme eingreifen und z. B. die bisher geleistete freiwillige Nachbarschaftshilfe durch Zeitgutschriften honorieren? Dazu wird eine komplexe Administration benötigt mit finanziellen Garantieversprechen über einen sehr langen Zeitraum. Mehr unter www.careum-weiterbildung.ch

(Careum Weiterbildung)

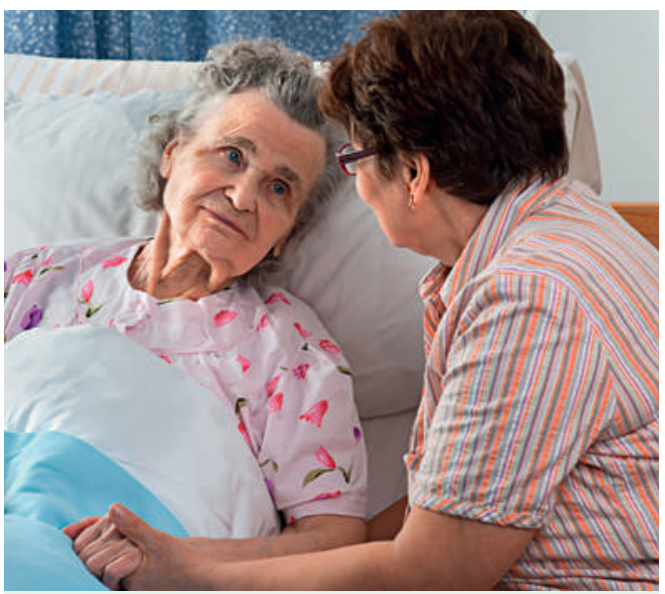

Die Idee: Noch nicht ganz Alte helfen schon ziemlich Alten und erhalten dafür Zeitgutschriften, die sie dann später als ziemlich Alte einlösen können. 